\title{
Cascading Plasmonic and Nonradiative Energy Transfer Interactions by Plasmon-Coupling Only Donor or Only Acceptor Quantum Dots of the Energy Transfer Pairs
}

\author{
Onur Akin ${ }^{1}$, Tuncay Ozel ${ }^{1}$, and Hilmi Volkan Demir ${ }^{1,2, *}$ \\ ${ }^{1}$ Department of Physics, Department of Electrical and Electronics Engineering, and UNAM - National Nanotechnology Research Center, \\ Bilkent University, TR-06800, Ankara, Turkey, ${ }^{2}$ Nanyang Technological University, School of Electrical \& Electronic Engineering, Division \\ of Microelectronics, School of Mathematical and Physical Sciences, Luminous! Semiconductor Lighting and Display Center of Excellence, \\ Nanyang Avenue, Singapore 639798, Singapore
}

Nonradiative energy transfer mechanism finds important applications in nanophotonics and nanobiology including nanoscale optical waveguiding and biological nanosensors. ${ }^{1,2}$ Various fluorophores can take part in such energy transfer interactions and their emission kinetics can thus be strongly modified. For example, colloidal semiconductor quantum dots, also known as nanocrystals (NCs), have widely been shown to serve as donors and acceptors among themselves or with other fluorescent species to transfer excitation energy nonradiatively in close proximity when the spectral conditions are set right. Emission characteristics of such fluorophores can be altered also when coupled with plasmonic structures, e.g., metal nanoparticles (MNPs), in their close proximity via strong plasmon-exciton interactions. One favored result of this plasmonic coupling mechanism is the spontaneous emission enhancement of NCs. Recently plasmon-mediated Förster-type resonance energy transfer (FRET) has been demonstrated and FRET rate has been reported to be increased between acceptor-donor pairs of different species (including NCs) that are both plasmon-coupled. ${ }^{3-5}$ In these previous reports, however, either donors and acceptors are blended and these blends are plasmon-coupled, or metal layer is placed between the donor and acceptor thin films to plasmon-couple both at the same time. In all of these prior works, the resulting plasmonexciton interactions are not controlled to take place either at the donor site or the acceptor site but at both of the sites. Therefore it has not been possible to identify the coupled interactions. Here we present the first proposition and demonstration of cascaded plasmonic and nonradiative energy transfer interactions that are controlled by selectively plasmon-coupling either only donor NCs or only acceptor NCs of the energy transfer pairs. This scheme uniquely allows for the ability to spatially control plasmon-exciton interactions to take place either at the "start" site (donors) or "finish" site (acceptors) of the energy transfer. This control is achieved by placing the plasmonic layer in the right proximity of the donors (for strong donor-exciton plasmon-coupling) while sufficiently being far away from the acceptors (for weak acceptor-exciton plasmon-coupling), or vice versa. Here we comparatively study and analyze consequent modifications of $\mathrm{NC}$ emission kinetics in response to both cases of plasmon-coupling to only the donor NCs and to only the acceptor NCs through steady-state and time-resolved photoluminescence measurements, along with their lifetime and rate calculations.
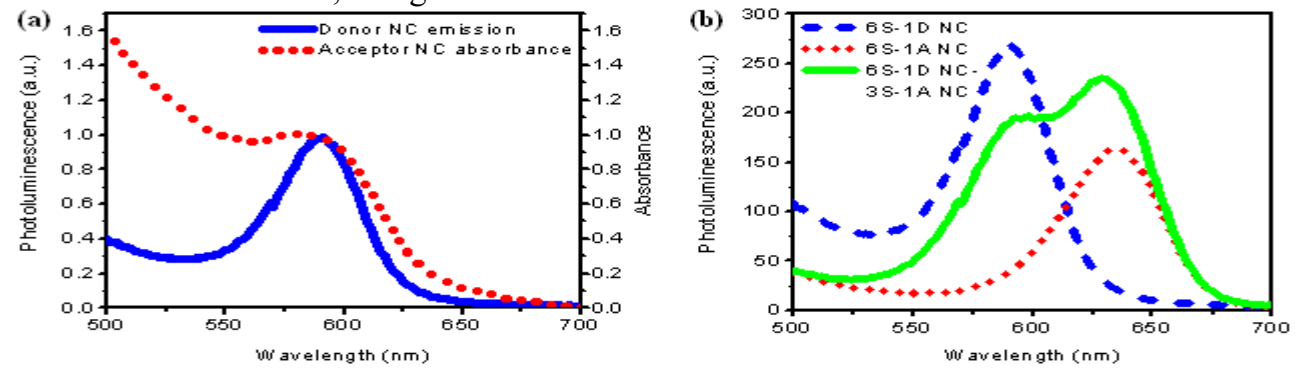

Figure 1. (a) Photoluminescence spectrum of donor CdTe NCs (solid blue) and optical absorption spectrum of acceptor CdTe NCs (dotted red). (b) Photoluminescence spectra of donor NCs (dashed blue: 6S-1DNC), acceptor NCs (dotted red: 6S-1ANC), and both NCs under FRET condition (solid green: 6S-1DNC-3S-1ANC).

We deposited monolayers (MLs) of NCs and MNP layers with nanometric precision via layer-by-layer selfassembly technique using a computerized dip-coating system. ${ }^{6}$ Gold (Au) nanoparticles (of 3-6 MLs) are laid down (to spectrally shift the plasmon resonance to a desired extend for the targeted plasmon-couplings). Also, donor (D) and acceptor (A) NCs made of CdTe are carefully synthesized in different sizes to tune the spectral overlap between donor emission and acceptor absorption as well as to match the plasmon resonance of MNPs. Following MNP deposition, 3-12 MLs of dielectric polyelectrolyte spacer (S) are coated and finally NC MLs are coated. As a negative control group we first study FRET between donor NCs and acceptor NCs in the absence of plasmonic structures. Strong spectral overlap between the absorption of acceptor NCs and the emission of donor NCs is satisfied for FRET condition, as depicted in Fig. 1(a). In this sample of 6S-1DNC-3S-1ANC, we observe a 1.43-fold emission enhancement of the acceptor NCs as a result of FRET (Fig. 1(b)). Also, the amplitude averaged photoluminescence lifetimes of the donor NCs decreases from 1.33 to 0.87 ns (due to energy transfer), while the 
lifetime of the acceptor NCs increases from 1.53 to $3.11 \mathrm{~ns}$ (because of energy feeding). Here the energy transfer rate is calculated to be $0.39 \mathrm{~ns}^{-1}$. Subsequently we repeat the same FRET experiment using plasmon-coupled acceptor NC film. As depicted in Fig. 2(a), there is a significant increase in the emission level of acceptor NC layer in the case of 6Au-6S-1ANC-3S-1DNC. Due to long separation, donor NCs are not influenced by the plasmonic resonance of Au NPs in this sample, as is separately confirmed experimentally. Here we observe a 2.86fold emission enhancement of the acceptor NCs (because of both plasmon-coupling and FRET this time). Timeresolved photoluminescence of this sample is depicted at the donor emission wavelength in Fig. 3(a). Since the donor NCs are not plasmon-coupled whereas the acceptor NCs are, the donor NC lifetime is not considerably modified and found to be $1.31 \mathrm{~ns}$, while the acceptor NC lifetime is substantially changed to $1.91 \mathrm{~ns}$. For this system, we calculate the energy transfer rate to be $0.37 \mathrm{~ns}^{-1}$. Finally we repeat FRET experiment with plasmoncoupling to only donor NCs. As depicted in Fig. 2(b), there is a significant increase in the emission levels of both donor and acceptor NCs this time for 6Au-6S-1DNC-3S-1ANC. Here we observe a 2.26-fold emission enhancement of the acceptor NCs (because of FRET), although there is no direct plasmon-coupling to these NCs, as is separately confirmed experimentally. Here the donor NC lifetime decreases down to $0.25 \mathrm{~ns}$ (due to both plasmon-coupling and energy transfer). For this system, we compute the energy transfer rate to be $0.49 \mathrm{~ns}^{-1}$.
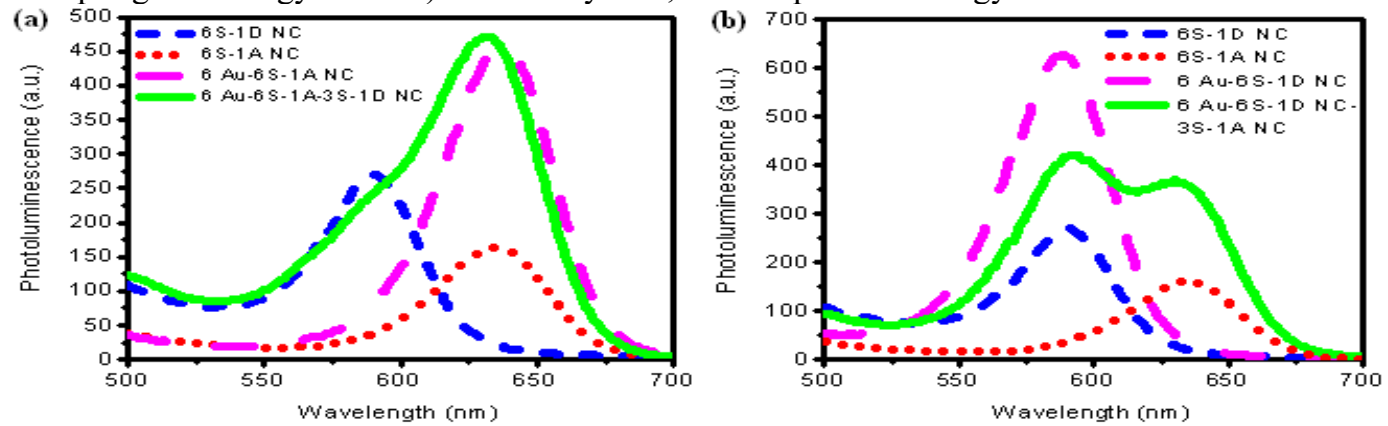

Figure 2. Photoluminescence spectra of (a) donor NCs (short dashed blue: 6S-1DNC), acceptor NCs (dotted red: 6S-1ANC), plasmon-coupled acceptor NCs in the presence of MNPs (long dashed pink: 6Au-6S-1ANC), and donor-acceptor NCs under FRET condition with plasmoncoupling to acceptor NCs (solid green: 6Au-6S-1 ANC-3S-1DNC) and (b) donor NCs (short dashed blue: 6S-1DNC), acceptor NCs (dotted red: 6S-1ANC), plasmon-coupled donor NCs in the presence of MNPs (long dashed pink: 6Au-6S-1DNC), and donor-acceptor NCs under FRET condition with plasmon-coupling to acceptor NCs (solid green: 6Au-6S-1DNC-3S-1 ANC)
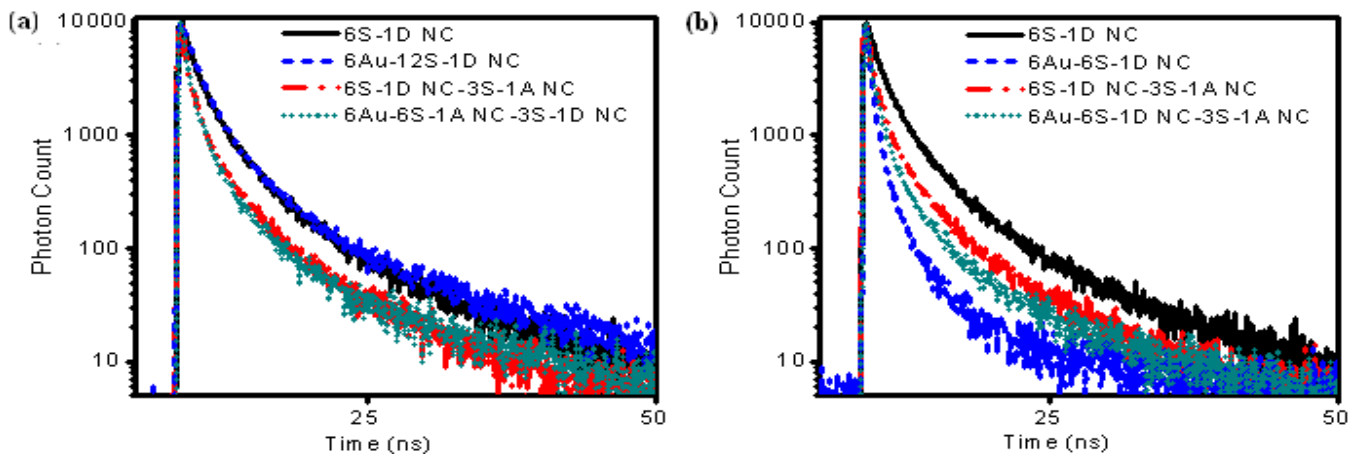

Figure 3. Time-resolved fluorescence decays, all at the donor emission peak wavelength, for (a) donor NCs (black: 6S-1D NC), donor NCs in the presence of MNPs that are far away (blue: 6Au-12S-1D NC), donor-acceptor NCs under FRET condition with no plasmon-coupling (red: 6S-1D-3S-1A NC) and donor-acceptor NCs under FRET condition with plasmon-coupling to acceptor NCs (green: 6Au-6S-1A-3S-1D NC) and (b) donor NCs (black: 6S-1D NC), donor NCs in the presence of MNPs that are close enough (blue: 6Au-6S-1D NC), donor-acceptor NCs under FRET condition with no plasmon-coupling (red: 6S-1D-3S-1A NC) and donor-acceptor NCs under FRET condition with plasmoncoupling to donor NCs (green: 6Au-6S-1ANC-3S-1DNC).

In conclusion, we proposed and demonstrated cascading of plasmonic and nonradiative energy transfer interactions via plasmon-coupling selectively either to only donor NCs or to only acceptor NCs of the energy transfer pairs. This approach allowed us to control spatially plasmon-exciton interactions to take place distinctly either at the energy transfer starting site of donors or at the energy transfer finishing site of acceptors.

This work is supported by NRF-RF 2009-09, EU-FP7 Nanophotonics4Energy NoE, BMBF TUR 09/001, and TUBITAK EEEAG 107E088, 109E002, 109E004, and 110E010. HVD acknowledges support from ESF-EURYI and TUBA-GEBIP, and OA, from TUBITAK-BIDEB.

References

[1] K. Matsuda,Y. Ito, Y. Kanemitsu, Appl. Phys Lett. 92, 211911 (2008). [4] X. Su et al. Appl. Phys. Lett. 96, 043106 (2010).

[2] V. K. Komarala et al., Appl. Phys Lett. 89, 253118 (2006). [5] C. H. Wang et al. Appl. Phys. Lett. 96,071906 (2010).

[3] O Kulakovich et al. Nano Lett. 2, 1449 (2002).

[6] G. Decher, Science 2775330 (1997). 